\title{
Relationship between Fragmented QRS Complex and Aortic Stiffness in Chronic Hemodialysis Patients
}

\author{
Aydın Güçlü ${ }^{a}$ Gökay Nar ${ }^{b}$ Atilla İçlib Nail Özhan ${ }^{c}$ Siren Sezer ${ }^{d}$ \\ Departments of ${ }^{a}$ Nephrology and ${ }^{b}$ Cardiology, Ahi Evran University Medical School, Kırşehir, ${ }^{c}$ Department of \\ Internal Medicine, Pamukkale University Medical School, Denizli, and ${ }^{\mathrm{d}}$ Department of Nephrology, Baskent \\ University Medical School, Ankara, Turkey
}

\section{Keywords}

Fragmented QRS complex · Aortic stiffness · Diastolic

dysfunction $\cdot$ Hemodialysis

\begin{abstract}
Objective: This study aimed to evaluate the correlation between fragmented QRS complex (fQRS), aortic stiffness, and diastolic dysfunction in hemodialysis patients. Subjects and Methods: A sample of 56 patients who received hemodialysis treatment was stratified into 2 groups according to their electrocardiography (ECG) patterns with or without fQRS. Baseline characteristics and laboratory parameters of patients were documented. Conventional echocardiographic and Doppler echocardiographic procedures were performed in all patients. The mean early (Em) diastolic and late (Am) diastolic myocardial velocities were calculated. These tests were performed before dialysis. The Student $t$ test, MannWhitney $U$ test, $X^{2}$ test, Spearman correlation, and multivariate linear regression analysis were used to analyze parameters where appropriate. Results: Of the 56 patients under hemodialysis, fQRS in ECG was detected in 26 (46.4\%). Echo-
\end{abstract}

cardiographic evaluation showed that deceleration time (237.57 $\pm 40.10 \mathrm{~ms} ; p=0.030)$, isovolumic relaxation time $(126.84 \pm 15.62 \mathrm{~ms} ; p<0.001)$, early $(\mathrm{E}) /$ late $(\mathrm{A})$ ventricular filling velocity $(\mathrm{E} / \mathrm{A})$ ratio $(1.15 \pm 0.40 ; p \leq 0.001)$, and aortic stiffness index value $(9.62 \pm 4.53 ; p=0.016)$ exhibited a statistical increase in hemodialysis patients with fQRS compared to patients without fQRS. E $(58.23 \pm 19.96 \mathrm{~m} / \mathrm{s} ; p=$ $0.004)$, and $\operatorname{Em}(5.96 \pm 2.08 \mathrm{~cm} / \mathrm{s} ; p=0.023)$ velocity levels were significantly lower in hemodialysis patients with fQRS than patients without fQRS. Aortic stiffness closely correlated with diastolic dysfunction (deceleration time $r=0.273$, $p=0.042$; isovolumic relaxation time $r=0.497, p<0.001 ; \mathrm{E} / \mathrm{A}$ ratio $r=-0.449, p=0.001)$. On multivariate linear regression analysis, fQRS and aortic stiffness were independently associated in hemodialysis patients $(\beta=0.321, p=0.049)$. Conclusions: Increased aortic stiffness and left ventricular systolic dysfunction were observed more frequently in hemodialysis patients with fQRS than in patients without fQRS. fQRS is an important determinant of aortic stiffness in hemodialysis patients.

C 2016 S. Karger AG, Basel

\begin{tabular}{|c|c|}
\hline KARGER & $\begin{array}{l}\text { Karger } \\
\text { Open access }\end{array}$ \\
\hline $\begin{array}{l}\text { E-Mail karger@karger.com } \\
\text { www.karger.com/mpp }\end{array}$ & $\begin{array}{l}\text { This is an Open Access article licensed under the terms of the } \\
\text { Creative Commons Attribution-NonCommercial } 3.0 \text { Un- } \\
\text { ported license (CC BY-NC) (www.karger.com/OA-license), } \\
\text { applicable to the online version of the article only. Distribu- } \\
\text { tion permitted for non-commercial purposes only. }\end{array}$ \\
\hline
\end{tabular}

Aydın Güclü, MD

Department of Nephrology, School of Medicine

Ahi Evran University

TR-40100 Kırșehir (Turkey)

E-Mail aydinguclu@gmail.com 


\section{Introduction}

Chronic kidney disease (CKD) is an independent risk factor for cardiovascular disease and is associated with increased cardiovascular morbidity and mortality [1]. Identification of patients at risk could alert physicians to monitor cardiovascular risk and implement the control of the aggressive risk factors.

A fragmented QRS complex (fQRS) is a bunch of electrocardiographic signals that show ventricular conduction delays in scarred myocardial regions [2]. The presence of fQRS complexes had been found to be associated with all-cause mortality and recurrent cardiac events [3]. fQRS had been shown as a highly sensitive and specific marker of myocardial scar [4].

Arterial stiffness is a measure of how the large arteries react to changes in blood pressure, and it is an important marker for endothelial dysfunction and atherosclerosis. Arterial stiffness increase is shown to be related with left ventricular (LV) diastolic dysfunction [5]. Aortic stiffness and LV diastolic dysfunction basically share a common etiological mechanism that includes tissue fibrosis. Myocardial fibrosis and scar result in deterioration of LV activities. It had been shown that inhomogeneous LV activity is related to fQRS formation in electrocardiography [6]. Korkmaz et al. [6] had shown the relation between aortic stiffness and fQRS in asymptomatic hypertensive patients. Although fQRS is known to be associated with increased cardiovascular morbidity and mortality, its role in predicting subclinical heart damage and effects on diastolic dysfunction and aortic stiffness has not been reported previously in hemodialysis patients. Hence, this study aimed to evaluate the relation among fQRS, diastolic dysfunction, and aortic stiffness in patients with CKD by using echocardiography and electrocardiography (ECG).

\section{Materials and Methods}

\section{Patient Selection}

Fifty-Six consecutive patients with CKD who had undergone hemodialysis treatment 3 times a week for at least 1 year in Ahi Evran University Medical Faculty from June 2014 to April 2015 were recruited into this study. Exclusion criteria were active infections, bundle-branch block (left bundle-branch block, incomplete or complete right bundle-branch block) or intraventricular conduction delay (duration of QRS $>120 \mathrm{~ms}$ ) on ECG, permanent pacemakers, significant stenosis by fractional flow reserve measurement, history of percutaneous or surgical revascularization, moderate to severe valve disease, LV dysfunction (ejection fraction $<40 \%$ ), liver failure, atrial fibrillation, congenital heart dis- ease, systemic conditions (ankylosing spondylitis, rheumatoid arthritis, Marfan and Ehlers-Danlos disease), and aortic aneurysms. The 56 patients were stratified into 2 groups according to their ECG patterns with (group 1; $n=26$ ) or without fQRS (group 2; $n=30$ ). Systolic and diastolic blood pressures were measured in each individual twice, following a 5-min rest, with an Erka brand sphygmomanometer using an appropriate cuff width. Fifteen patients who were on hypertension medication were recorded. The protocol was approved by the institutional review boards of the Faculty of Medicine, Pamukkale University, Denizli, Turkey, and adhered to the Declaration of Helsinki. Patients were informed about the study, and written consent was obtained from all subjects.

\section{Biochemical Analysis}

A 5-mL blood sample was taken just before hemodialysis treatment following $12 \mathrm{~h}$ of fasting. Complete blood count measurement was made using flow cytometry, fasting blood glucose, creatinine, albumin, and serum lipid measurements with an enzymatic colorimetric method, C-reactive protein measurements were done using an immunoturbidimetric method, and sodium, potassium, and chloride measurements were made with an ionselective electrode method. A Sysmex XT 2000I device was used for complete blood count, and the other biochemical parameters were studied using the Modular P Roche/Hitachi device.

\section{Measurement of $f Q R S$}

fQRS was defined as presence of at least 1 of the following findings in 12-lead routine ECG before dialysis: presence of an additional $\mathrm{R}$ wave (R'), notching of R or S wave, or $>1$ R' fragmentation in 2 contiguous leads [7]. The ECGs were analyzed by 2 independent cardiologists (G.N., A.İ.) blinded to the patient characteristics.

\section{Echocardiographic Data}

Echocardiography was performed in the patients approximately $1 \mathrm{~h}$ before dialysis after a $15-\mathrm{min}$ rest. The entire study population of the 56 patients underwent screening by 2-dimensional echocardiography, which consisted of conventional Doppler sonography via a standard 2-dimensional transducer device (Vingmed System Vivid 5; GE Vingmed Ultrasound, Horten, Norway); images were obtained in the left lateral decubitus position. LV filling was evaluated by pulse wave Doppler sonography, from the apical 4-chamber view, with the sample volume positioned at the tips of the mitral valve. We recorded the early (E)/late (A) ventricular filling velocity (E/A) ratio, isovolumic relaxation time (IVRT), and E wave deceleration time (DT), together with early and late diastolic velocities. Tissue Doppler imaging utilizes modified wall filter and reduced gain in order to display myocardial velocity while avoiding blood flow detection. From the 4 -chamber view, the tissue Doppler imaging sample volume was placed sequentially at septal and lateral sides of the valvular ring. The mean early (Em) diastolic, late diastolic, and systolic myocardial velocities were calculated $(\mathrm{cm} / \mathrm{s})$. Furthermore, we measured the $\mathrm{E} / \mathrm{Em}$ ratio. All measurements were done at $50 \mathrm{~m} / \mathrm{s}$ at the end of the expiration. The modified Simpson method was used to calculate LV ejection fraction.

The M-mode rod was placed so that it could pass through the aortic region that was $3 \mathrm{~cm}$ distal to the aortic valve. Systolic and diastolic diameters of the ascending aorta were obtained from the 
Table 1. Comparison of biochemical features of the hemodialysis patients

\begin{tabular}{lccc}
\hline & Group 1 & Group 2 & $p$ value \\
\hline Leukocytes, $n / \mathrm{mm}^{3}$ & $7.61 \pm 2.82$ & $7.92 \pm 3.51$ & 0.764 \\
Hemoglobin, g/dL & $10.45 \pm 1.71$ & $11.07 \pm 1.60$ & 0.233 \\
Urea, mg/dL & $151.63 \pm 35.78$ & $158.33 \pm 29.05$ & 0.800 \\
Creatinine, mg/dL & $8.33 \pm 2.21$ & $8.07 \pm 2.54$ & 0.722 \\
Albumin, mg/dL & $3.50 \pm 0.27$ & $3.56 \pm 0.28$ & 0.561 \\
Uric acid, mg/dL & $6.48 \pm 1.00$ & $6.40 \pm 1.18$ & 0.818 \\
Glucose, mg/dL & $108.30 \pm 40.05$ & $126.70 \pm 48.59$ & 0.140 \\
Ferritin, mg/dL & $1,013.45 \pm 410.09$ & $802.10 \pm 404.79$ & 0.109 \\
Total cholesterol, & & & \\
$\quad$ & $169.09 \pm 38.60$ & $174.90 \pm 41.88$ & 0.638 \\
LDL, mg/dL & $98.63 \pm 27.22$ & $105.95 \pm 26.86$ & 0.387 \\
HDL, mg/dL & $34.41 \pm 9.10$ & $34.57 \pm 8.71$ & 0.953 \\
Triglyceride, mg/dL & $180.31 \pm 83.28$ & $191.66 \pm 87.62$ & 0.683 \\
Calcium, mg/dL & $9.16 \pm 0.74$ & $8.80 \pm 0.60$ & 0.887 \\
Phosphorus, mg/dL & $4.94 \pm 1.29$ & $4.71 \pm 1.37$ & 0.580 \\
CRP, mg/dL & $2.63 \pm 3.70$ & $1.64 \pm 2.61$ & 0.320 \\
PTH, pg/mL & $383.68 \pm 441.61$ & $217.95 \pm 143.41$ & 0.119 \\
UF, mL & $2,938.88 \pm 675.74$ & $2,984.21 \pm 857.81$ & 0.860 \\
Duration of chronic & & & \\
$\quad$ renal failure, months & $90.78 \pm 67.96$ & $74.28 \pm 47.18$ & 0.359 \\
Kt/V & $1.49 \pm 0.30$ & $1.47 \pm 0.18$ & 0.800 \\
\hline & & & \\
\hline
\end{tabular}

Group 1: hemodialysis patients with fQRS; group 2: hemodialysis patients without fQRS; LDL, low-density lipoprotein; HDL, high-density lipoprotein; CRP, C-reactive protein; PTH, parathyroid hormone; UF, amount of liquid drawn in a single dialysis session; $\mathrm{Kt} / \mathrm{V}$, product of dialyzer urea clearance and treatment time divided by body urea volume.

aortic trace. Systolic diameter was measured from the location of the aortic trace in which the maximum forward movement was observed, while diastolic diameter was measured from the location that corresponded to the R spike of the ECG. To determine the pulse pressure, which was necessary for the calculation of relevant parameters, the systolic blood pressure and diastolic blood pressure were concurrently measured with a mercury sphygmomanometer. The difference between the 2 blood pressure values was accepted as the pulse pressure. Average systolic and diastolic measurements were calculated following 3 consecutive measurements. The aortic elastic properties were calculated by using the formulae below:

Aortic strain $=\frac{\text { systolic aortic diameter }- \text { diastolic aortic diameter }}{\text { diastolic diameter }} \times 100$
Distensibility $\left(\mathrm{cm}^{2} \times \mathrm{dyn}^{-1}\right)=\frac{2 \times(\text { aortic strain })}{\text { systolic pressure }- \text { diastolic pressure }}$
Aortic stiffness index $=\frac{\ln \left(\frac{\text { systolic blood pressure }}{\text { diastolic blood pressure }}\right) \times \text { aortic diastolic diameter }}{(\text { aortic systolic diameter }- \text { aortic diastolic diameter })}$.

68

Med Princ Pract 2017;26:66-70

DOI: $10.1159 / 000452418$
Table 2. Comparison of the clinical and demographic data in hemodialysis patients

\begin{tabular}{lccc}
\hline & Group 1 & Group 2 & $\begin{array}{l}p \\
\text { value }\end{array}$ \\
& & & \\
\hline Etiology of CKD, \% & & & \\
Diabetes mellitus & 42.3 & 36.7 & 0.437 \\
Hypertension & 23.1 & 30.0 & 0.391 \\
Glomerulonephritis & 7.7 & 10.0 & 0.569 \\
Nephrolithiasis & 3.8 & 3.3 & 0.718 \\
Unknown & 22.2 & 17.8 & 0.788 \\
\hline Medications, \% & & & \\
Erythropoietin & 38.5 & 36.7 & 0.94 \\
Vitamin D & 26.9 & 33.3 & 0.452 \\
3-Blockers & 11.5 & 20.0 & 0.383 \\
ACE/ARB & 0.0 & 3.3 & 0.253 \\
Antilipidemic agent & 69.2 & 3.3 & 0.536 \\
Ca-based phosphate binders & 15.5 & 56.7 & 0.245 \\
ACE & 50.0 & 20 & 0.560 \\
\hline Demographics & & & \\
Gender male, \% & 42.3 & 53.6 & 0.508 \\
Age, years & $59.82 \pm 13.98$ & $66.23 \pm 11.10$ & 0.102 \\
\hline
\end{tabular}

Group 1: hemodialysis patients with fQRS; group 2: hemodialysis patients without fQRS; CKD, chronic kidney disease; ACE, angiotensin-converting enzyme inhibitors; $\mathrm{ARB}$, angiotensin receptor blockers.

\section{Statistical Analysis}

The continuous variables are expressed as means $\pm \mathrm{SD}$, whereas categorical variables are percentages. $p<0.05$ was statistically significant. Parametric continuous variables were compared by the independent Student $t$ test or Mann-Whitney $U$ test. Categorical variables were analyzed by the $\chi^{2}$ and Fisher exact tests. Spearman correlation was used for correlation analysis. Multiple regression analysis was made for the relation between fQRS and aortic stiffness. The statistical analyses were carried out with the Windows SPSS v17.0 package program.

\section{Results}

There were no significant differences between patients with $\mathrm{fQRS}$ and without $\mathrm{fQRS}$ when they were evaluated for baseline characteristics such as etiology of CKD, medications, gender and age (Table 1), and laboratory parameters $(p>0.05$; Table 2$)$.

Echocardiographic evaluation showed that diastolic parameters like early diastolic velocity $(58.23 \pm 19.96 \mathrm{~m} / \mathrm{s}$; $p=0.004)$, and $\operatorname{Em}(5.96 \pm 2.08 \mathrm{~cm} / \mathrm{s} ; p=0.023)$ levels were significantly low in hemodialysis patients with fQRS. DT $(237.57 \pm 40.10 \mathrm{~ms} ; p=0.030)$, IVRT $(p<0.001)$, and E/A ratio $(1.15 \pm 0.40 ; \mathrm{p}<0.001)$ values exhibited a statistical 
Table 3. The measurement of echocardiographic parameters in groups 1 and 2

\begin{tabular}{lcrc}
\hline & Group 1 & \multicolumn{1}{l}{ Group 2 } & $p$ value \\
\hline LVEDD, mm & $46.30 \pm 5.76$ & $47.30 \pm 5.25$ & 0.504 \\
LVESD, mm & $31.26 \pm 6.36$ & $32.06 \pm 5.87$ & 0.628 \\
IVS thickness, mm & $15.03 \pm 10.19$ & $112.66 \pm 1.76$ & 0.215 \\
PW thickness, mm & $12.96 \pm 1.75$ & $4,912.66 \pm 1.72$ & 0.530 \\
LVEF, \% & $57.11 \pm 10.75$ & $58.13 \pm 7.58$ & 0.681 \\
LA dimension, mm & $41.88 \pm 5.33$ & $40.30 \pm 5.01$ & 0.257 \\
Aortic diastolic & & & \\
$\quad$ diameter, mm & $30.02 \pm 4.18$ & $30.01 \pm 4.59$ & 0.998 \\
E, m/s & $58.23 \pm 19.96$ & $78.83 \pm 29.74$ & 0.004 \\
A, m/s & $77.76 \pm 17.34$ & $73.03 \pm 28.38$ & 0.463 \\
DT, ms & $237.57 \pm 40.10$ & $212.73 \pm 43.73$ & 0.030 \\
IVRT, ms & $126.84 \pm 15.62$ & $107.30 \pm 17.89$ & 0.000 \\
Em, cm/s & $5.96 \pm 2.08$ & $7.63 \pm 3.06$ & 0.023 \\
E/A ratio & $1.15 \pm 0.40$ & $0.76 \pm 0.26$ & 0.000 \\
\hline
\end{tabular}

Group 1: hemodialysis patients with fQRS; group 2: hemodialysis patients without fQRS; LVEDD, left ventricular end-diastolic dimension; LVESD, left ventricular end-systolic dimension; IVS, ventricular septum; PW, posterior wall; LVEF, left ventricular ejection fraction; LA, left atrium; E, early diastolic flow; A, atrial contraction signal; DT, deceleration time; IVRT, isovolumic relaxation time.

Table 4. The measurement of aortic elastic properties in groups 1 and 2

\begin{tabular}{llll}
\hline & Group 1 & Group 2 & $p$ value \\
\hline Aortic strain, \% & $5.66 \pm 2.38$ & $7.64 \pm 2.84$ & 0.007 \\
Distensibility, $\mathrm{cm}^{2} \times \mathrm{dyn}^{-1}$ & $2.63 \pm 1.05$ & $3.58 \pm 1.91$ & 0.029 \\
Aortic stiffness index & $9.62 \pm 4.53$ & $7.02 \pm 3.23$ & 0.016 \\
\hline
\end{tabular}

Group 1: hemodialysis patients with fQRS; group 2: hemodialysis patients without fQRS.

increase in hemodialysis patients with fQRS (Table 3). There was a significant difference between the groups, and aortic elastic properties (Table 4), aortic strain (5.66 $\pm 2.38 \%)$, and distensibility $\left(2.63 \pm 1.05 \mathrm{~cm}^{2} \times\right.$ dyn $\left.^{-1}\right)$ were significantly lower in the fQRS group $(p<0.01)$ patients. The aortic stiffness index $(9.62 \pm 4.53)$ was significantly higher in the fQRS group $(\mathrm{p}<0.01)$.

In this study, a statistically significant relationship was identified between aortic stiffness and diastolic dysfunction in hemodialysis patients (DT $r=0.273, p=0.042$; IVRT $r=0.497, p<0.001$; E/A ratio $r=-0.449, p=0.001$ ).

Fragmented QRS Complex in

Hemodialysis Patients
After adjusting the C-reactive protein value, disease duration of CKD and albumin in multiple regression analysis, fQRS was significantly correlated independently with aortic stiffness $(\beta=0.313, p=0.048 ; 95 \%$ confidence interval: 0.19-5.33).

\section{Discussion}

In this study, aortic stiffness increase and LV diastolic dysfunction were more common in hemodialysis patients with $f Q R S$ than patients without fQRS. fQRS and aortic stiffness were independently associated in hemodialysis patients. Also, it was shown that aortic stiffness increase was related to LV diastolic dysfunction.

The finding that LV diastolic dysfunction was severer in hemodialysis patients with fQRS than patients without fQRS confirmed that of Ulusoy et al. [8] who had demonstrated that fQRS was related with systolic and diastolic dysfunction of the left ventricle in kidney transplant patients. Also Adar et al. [9] showed that fQRS was independently associated with subclinical LV dysfunction in patients with CKD and normal ejection fraction. A probable cause of the relationship between diastolic function and fQRS could be myocardial fibrosis causing inhomogeneous activation and $\mathrm{fQRS}$ formation that causes diastolic dysfunction [8-10].

In this study the finding that an independent relationship exists between fQRS and aortic stiffness confirmed that the presence of fQRS reported by Korkmaz et al. [6] is associated independently with increased arterial stiffness in asymptomatic hypertensive patients. However, Ikonomidis et al. [11] reported the relationship between diastolic dysfunction and aortic distensibility in hypertensive patients, and also LV mass and function were reported to be related to aortic stiffness in previous studies $[12,13]$. There are possible explanations for the relation between aortic distensibility and diastolic dysfunction. LV diastolic dysfunction and aortic stiffness may be epiphenomena of the myocardial and aortic injuries in hemodialysis patients [14]. The second possible explanation is that increased aortic stiffness may also increase afterload. An increase in afterload induces myocardial structural changes of the left ventricle and thus causes LV diastolic dysfunction [15]. Additionally, structural changes in left ventricle were suggested to be related with aortic stiffness [15-17].

Age is the main clinical marker in arterial stiffness. Aortic stiffness is identified to be $5-10 \%$ less in women. Also blood pressure and diabetes mellitus can affect aor-

Med Princ Pract 2017;26:66-70 
tic stiffness in kidney disease [18]. We did not observe any statistically significant differences between patients with and without fQRS in terms of age, gender, diabetes mellitus, blood pressure levels, chronic kidney failure duration and $\mathrm{Kt} / \mathrm{V}$ value which shows adequateness of dialysis.

\section{References}

1 Lai S, Dimko M, Galani A, et al: Early markers of cardiovascular risk in chronic kidney disease. Ren Fail 2015;37:254-261.

2 Das MK, Michael MA, Suradi H, et al: Usefulness of fragmented QRS on a 12-lead electrocardiogram in acute coronary syndrome for predicting mortality. Am J Cardiol 2009;104: 1631-1637.

3 Das MK, Saha C, El Masry H, et al: Fragmented QRS on a 12-lead ECG: a predictor of mortality and cardiac events in patients with coronary artery disease. Heart Rhythm 2007;4: 1385-1392.

4 Russo C, Jin Z, Palmieri V, et al: Arterial stiffness and wave reflection: sex differences and relationship with left ventricular diastolic function. Hypertension 2012;60:362-368.

5 Flowers NC, Horan LG, Thomas JR, et al: The anatomic basis for high-frequency components in the electrocardiogram. Circulation 1969;39:531-539.

6 Korkmaz L, Hatem E, Erkan H, et al: Fragmented QRS may predict increased arterial stiffness in asymptomatic hypertensive patients. Blood Press Monit 2015;20:16-19.

7 Tyralla K, Amann K: Morphology of the heart and arteries in renal failure. Kidney Int 2003; 63:80-83.

\section{Conclusion}

In this study increased aortic stiffness and LV systolic dysfunction were observed more frequently in hemodialysis patients with fQRS than patients without fQRS. fQRS was related independently with aortic stiffness. Presence of $\mathrm{fQRS}$ can be used for risk stratification in hemodialysis patients.
8 Ulusoy S, Ozkan G, Adar A, et al: Relationship between fragmented QRS complex and left ventricular systolic and diastolic function in kidney transplant patients. Prog Transplant 2014;24:146-151

9 Adar A, Kiriş A, Ulusoy S, Ozkan, et al: Fragmented QRS is associated with subclinical left ventricular dysfunction in patients with chronic kidney disease. Acta Cardiol 2014;69: 385-390.

10 Mahenthiran J, Khan BR, Sawada SG, et al: Fragmented QRS complexes not typical of a bundle branch block: a marker of greater myocardial perfusion tomography abnormalities in coronary artery disease. J Nucl Cardiol 2007; 14:347-353.

11 Ikonomidis I, Tzortzis S, Papaioannou T, et al: Incremental value of arterial wave reflections in the determination of left ventricular diastolic dysfunction in untreated patients with essential hypertension. J Hum Hypertens 2008;22:687-698.
12 Benetos A, Waeber B, Izzo J, et al: Influence of age risk factors and cardiovascular and renal disease on arterial stiffness: clinical applications. Am J Hypertens 2002;15:1101-1108.

13 Palmieri V, Bella JN, Roman MJ, et al: Pulse pressure/stroke index and left ventricular geometry and function: the LIFE Study. J Hypertens 2003;21:781-787.

14 Vinereanu D, Nicolaides E, Boden L, et al: Conduit arterial stiffness is associated with impaired left ventricular subendocardial function. Heart 2003;89:449-450.

15 Roman MJ, Ganau A, Saba PS, et al: Impact of arterial stiffening on left ventricular structure. Hypertension 2000;36:489-494.

16 Ren JF, Pancholy SB, Iskandrian AS, et al: Doppler echocardiographic evaluation of the spectrum of left ventricular diastolic dysfunction in essential hypertension. Am Heart J 1994;127:906-913.

17 Das MK, Khan B, Jacob S, et al: Significance of a fragmented QRS complex versus a $Q$ wave in patients with coronary artery disease. Circulation 2006;113:2495-2501.

18 Pluta A, Stróżecki P, Krintus M, et al: Left ventricular remodeling and arterial remodeling in patients with chronic kidney disease stage 1-3. Ren Fail 2015;9:1-6. 